\title{
Kinetic Models of Solar Wind Electrons, Protons and Heavy lons
}

\author{
Viviane Pierrard \\ Belgian Institute for Space Aeronomy and Université Catholique de Louvain \\ Belgium
}

\section{Introduction}

In the present chapter, we describe the recent results obtained in the development of solar wind models using the kinetic approach. We show how the solution of the evolution equation is used to determine the velocity distribution function (VDF) of the solar wind particles and their moments. The solutions depend on the approximations and assumptions made in the development of the models. We describe in particular the results obtained with the collisionless exospheric approximation, the effects of Coulomb collisions obtained by using a Fokker-Planck term in the evolution equation, the effects of wave turbulence for the electrons and those of kinetic Alfven waves for the protons.

\section{The kinetic approach}

Solar wind models have been developed on the basis of the magnetohydrodynamic (MHD) and of the kinetic approaches. These two theories are complementary, but we will here emphasize the recent advances in the kinetic solar wind representation

Kinetic models provide the velocity distribution functions $\mathrm{f}(\vec{r}, \vec{v}, \mathrm{t})$ of the particles as a solution of the evolution equation:

$$
\frac{\partial f}{\partial t}+\vec{v} \cdot \frac{\partial f}{\partial \vec{r}}+\vec{a} \cdot \frac{\partial f}{\partial \vec{v}}=\left(\frac{d f}{d t}\right)_{c}
$$

where the first term represents the time dependence of the VDF, the second term corresponds to the spatial diffusion ( $\vec{r}$ is the position and $\vec{v}$ the velocity vector of the particles), the third term takes into account the effects of the external forces $\vec{F}(\vec{a}=\vec{F} / m$ where $\vec{a}$ is the acceleration and $m$ is the mass of the particles), and the term in the right hand side of the equation represents the effects of collisions and other interactions.

The calculation of the VDF moments gives the macroscopic quantities such as the number density:

$$
n(\vec{r})=\int_{-\infty}^{\infty} f(\vec{r}, \vec{v}) d \vec{v}
$$


the particle flux:

$$
\vec{F}(\vec{r})=\int_{-\infty}^{\infty} f(\vec{r}, \vec{v}) \vec{v} d \vec{v}
$$

the bulk velocity:

$$
\vec{u}(\vec{r})=\frac{\vec{F}(\vec{r})}{n(\vec{r})}
$$

the pressure:

$$
\vec{P}(\vec{r})=m \int_{-\infty}^{\infty} f(\vec{r}, \vec{v})(\vec{v}-\vec{u})(\vec{v}-\vec{u}) d \vec{v}
$$

the temperature:

$$
T(\vec{r})=\frac{m}{3 k n(\vec{r})} \int_{-\infty}^{\infty} f(\vec{r}, \vec{v})|v-u|^{2} d \vec{v}
$$

the energy flux.

$$
\vec{E}(\vec{r})=\frac{m}{2} \int_{-\infty}^{\infty} f(\vec{r}, \vec{v})|v-u|^{2}(\vec{v}-\vec{u}) d \vec{v}
$$

We are interested by the steady state solutions of the evolution equation, i.e., we consider $\partial f / \partial t=0$.

\section{Exospheric models}

The simplest approximation in low density plasmas like the solar corona and the solar wind where kinetic processes prevail is to consider that there are no interactions at all between the particles above a certain radial distance called the exobase. In this collisionless region located already at low radial distances in the solar corona (the exobase is typically located between 1.1 Rs and $6 \mathrm{Rs}$ ), one considers the exospheric approximation: the right hand side term in eq. (1) is neglected, i.e., we assume $(\mathrm{df} / \mathrm{dt})_{\mathrm{c}}=0$. The particles are only submitted to the effects of the external forces, i.e., the gravitational force, the electric force and the Lorentz force due to the presence of the magnetic field $\vec{B}$ :

$$
\vec{a}=\left(\vec{g}+\frac{Z e \vec{E}}{m}\right)+\frac{Z e}{m}(\vec{v} \times \vec{B})
$$

The Vlasov equation can then be solved analytically assuming an electric force that ensures the equality of electron and ion fluxes. If the VDF of the particles is known at the reference level, the solution of the Vlasov equation determines the VDF of the particles at the other radial distances. Such a model was developed by Pierrard \& Lemaire (1996) for the ion-exosphere and adapted by Maksimovic et al. (1997a) and later by Lamy et al. (2003) for the solar wind. 


\subsection{The electrons}

In exospheric models, it is assumed that the energy and the magnetic moment are conserved. The trajectories of the particles depend on their velocity and pitch angle. The electrons are in an attractive potential due to the attractive electric and the gravitational potentials, so that 4 different orbits are then possible:

- Ballistic particles: these electrons have not enough energy to escape, so they come back to the Sun. The low energy part of the electron VDF is thus symmetric.

- $\quad$ Escaping particles: these electrons have enough energy to escape.

- Trapped particles: these electrons are trapped by the magnetic force. They can not escape and give also a symmetric part in the VDF.

- Incoming particles: these electrons correspond to particles coming from the interplanetary space to the Sun.

These different orbits are illustrated on Fig. 1 in the velocity plane parallel and perpendicular to the magnetic field.

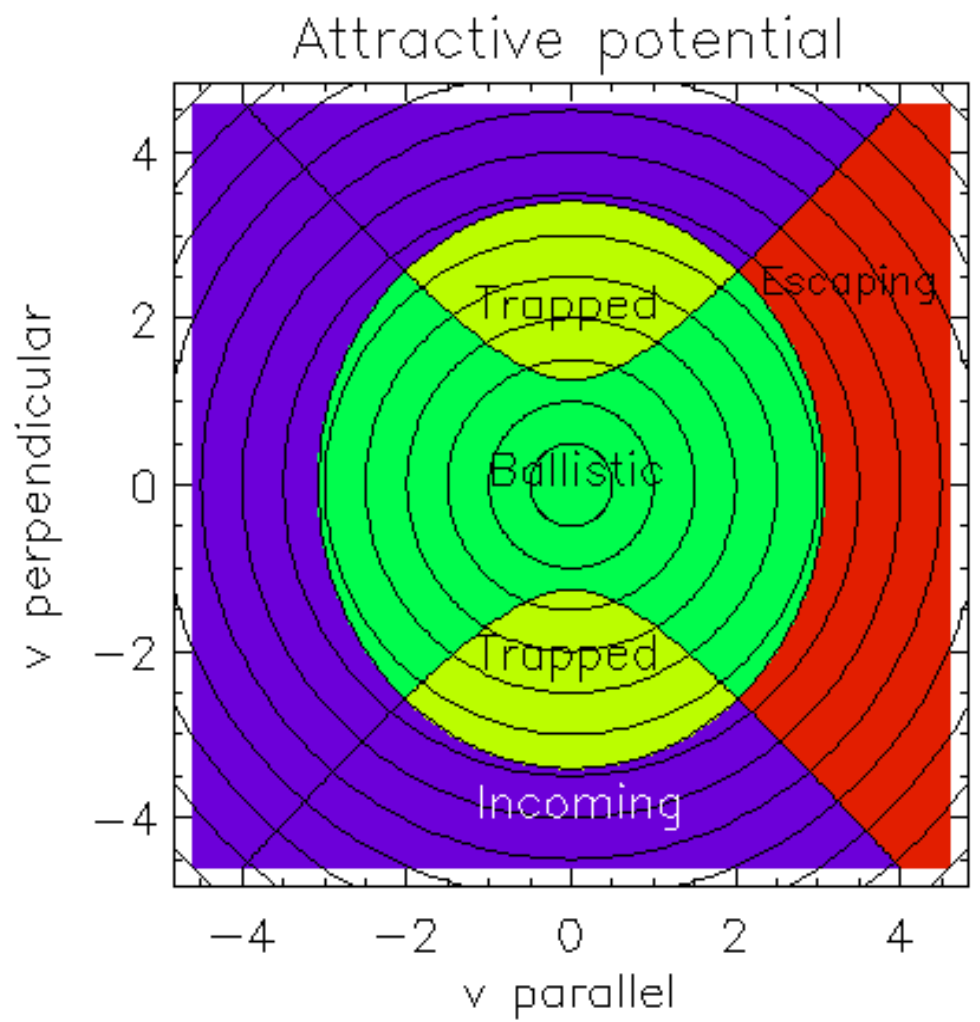

Fig. 1. Isocontours of the VDF of particles in an attractive potential like the solar wind electrons (circles). The 4 different classes of the particles (ballistic, escaping, trapped and incoming) determined by the energy and magnetic moment conservation laws are illustrated in different colours. 
In exospheric models, it is assumed that there are no particles incoming from the interplanetary space so that the distribution is anisotropic. The escape flux is thus only contributed by energetic electrons. Indeed, only energetic electrons can pass over the potential barrier. The VDF is thus a truncated anisotropic distribution that leads to non-zero external flux and heat flux.

\subsection{The protons}

The protons are on the contrary in a repulsive potential, because the repulsive electrostatic potential exceeds the attractive gravitational potential at sufficiently large radial distances. There are then neither ballistic nor trapped orbits. Assuming again an empty population of incoming particles flying from the interplanetary space to the Sun, there are only escaping protons that are accelerated outwards in the solar wind by the electric force. When the exobase is located at very low radial distance like in the coronal holes, the potential can first be attractive at low radial distances and then become repulsive above a radial distance called $r_{\max }$ that is located at a few solar radii. The global proton potential shows then a maximum at low radial distance (Lamy et al., 2003). The different orbits of the solar wind protons in a repulsive potential located after $r_{\max }$ are illustrated on Fig. 2.

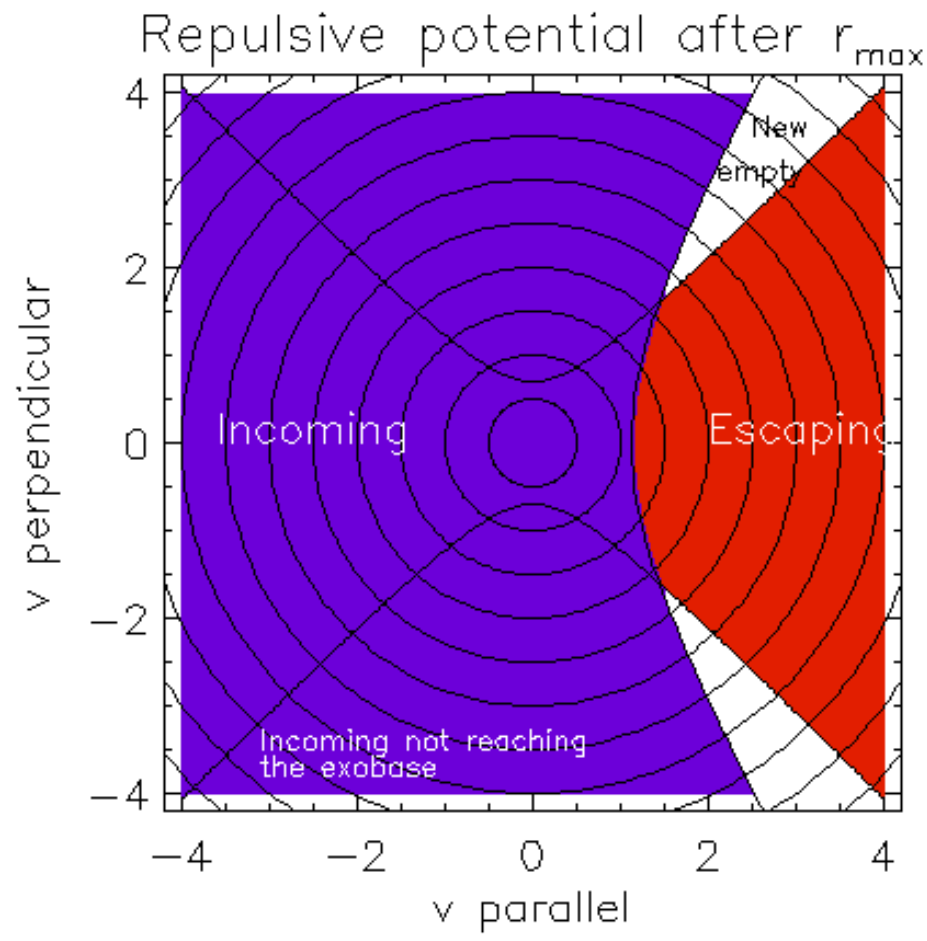

Fig. 2. Isocontours of the VDF of particles (circles) in a repulsive potential like the solar wind protons at large radial distances. The 2 different classes of particles (escaping and incoming) determined by the energy and magnetic moment conservation laws are illustrated in different colours. 


\subsection{The profiles of the moments}

When the VDF of the particles is known at any radial distances, one can calculate the moments by eq. (2) to (6). Fig. 3 shows typical radial profiles of the solar wind obtained with an exospheric model assuming that at the reference exobase level at $1.1 \mathrm{Rs}$, the temperature of the electrons $T_{e}=810^{5} \mathrm{~K}$ and of the protons $T_{p}=10^{6} \mathrm{~K}$. The maximum of the proton potential at $r_{\max }$ is well visible on panel 7 of Fig. 3.

The exospheric models have established that the physical process implicated in the solar wind acceleration is the electric field that pushes the positive ions outwards (Lemaire \& Pierrard, 2001). This electric field is increased when an enhanced population of suprathermal electrons is assumed to be present in the solar corona (Pierrard \& Lemaire, 1996; Maksimovic et al., 2001). This can be modeled by assuming for instance a Kappa distribution for the electrons. This distribution decreases as a power law of the energy.

Electron VDFs measured in situ in the solar wind are characterized by a thermal core population and a halo of suprathermal electrons (Pierrard et al., 2001b). Such distributions with suprathermal tails are well fitted by the so-called Kappa or Lorentzian distributions. The value of the kappa index determines the slope of the energy spectrum of the suprathermal electrons forming the tail of the VDF. In the limit $\kappa \rightarrow \infty$, the Kappa function degenerates into a Maxwellian. The kappa values obtained by fitting the observed electron VDF from Ulysses in the high speed solar wind range between 2 and 7 (Maksimovic et al., 1997b). The Kappa fit parameters obtained in the slow speed solar wind are a little bit larger than in the high speed solar wind, confirming empirically a link between the velocity and the suprathermal electrons. The presence of similar power law distributions in many different space plasmas suggests a universal mechanism for the creation of such suprathermal tails (Pierrard \& Lazar, 2010 for a review).

The electrostatic potential found in this example with an assumed kappa value of $\kappa=5$ for the electron VDF is illustrated on Panel 2 of Fig. 3. Note that contrary to the electrons, the proton distribution is assumed to be Maxwellian in the corona. Anyway, suprathermal protons have no significant influence on the final bulk velocity of the solar wind, contrary to the suprathermal electrons that are the only one to be able to escape and are thus crucial in the determination of the electric potential.

Figure 3 shows typical radial profiles of the solar wind obtained with the exospheric model assuming a moderate value of Kappa $(\kappa=5)$ for the electrons at the exobase level $1.1 \mathrm{Rs}$ where the temperature of the electrons is assumed to be $T_{\mathrm{e}}=810^{5} \mathrm{~K}$ and that of the protons $T_{p}=10^{6} \mathrm{~K}$. This case gives a final bulk velocity at $215 \mathrm{Rs}$ of $301 \mathrm{~km} / \mathrm{s}$ (see panel 4) and a potential difference of $1250 \mathrm{~V}$ (see panel 2). This corresponds well to the slow speed solar wind that originates from equatorial streamers during minimum solar activity.

Smaller values of kappa (thus more suprathermal electrons) give larger potential differences and thus larger final bulk velocities at $1 \mathrm{AU}$, as observed in the high speed solar wind where the average velocity is around $800 \mathrm{~km} / \mathrm{s}$. With kappa=2 and the same other values for the temperatures at the exobase as in the example given Fig. 3, a bulk velocity as high as 1419 $\mathrm{km} / \mathrm{s}$ is obtained at $1 \mathrm{AU}$. On the contrary, when $\kappa$ tends to infinity, a simple Maxwellian distribution is recovered for the electron distribution and the bulk velocity is limited to 163 $\mathrm{km} / \mathrm{s}$. The most realistic velocities in the model, compared to what is observed in the high 

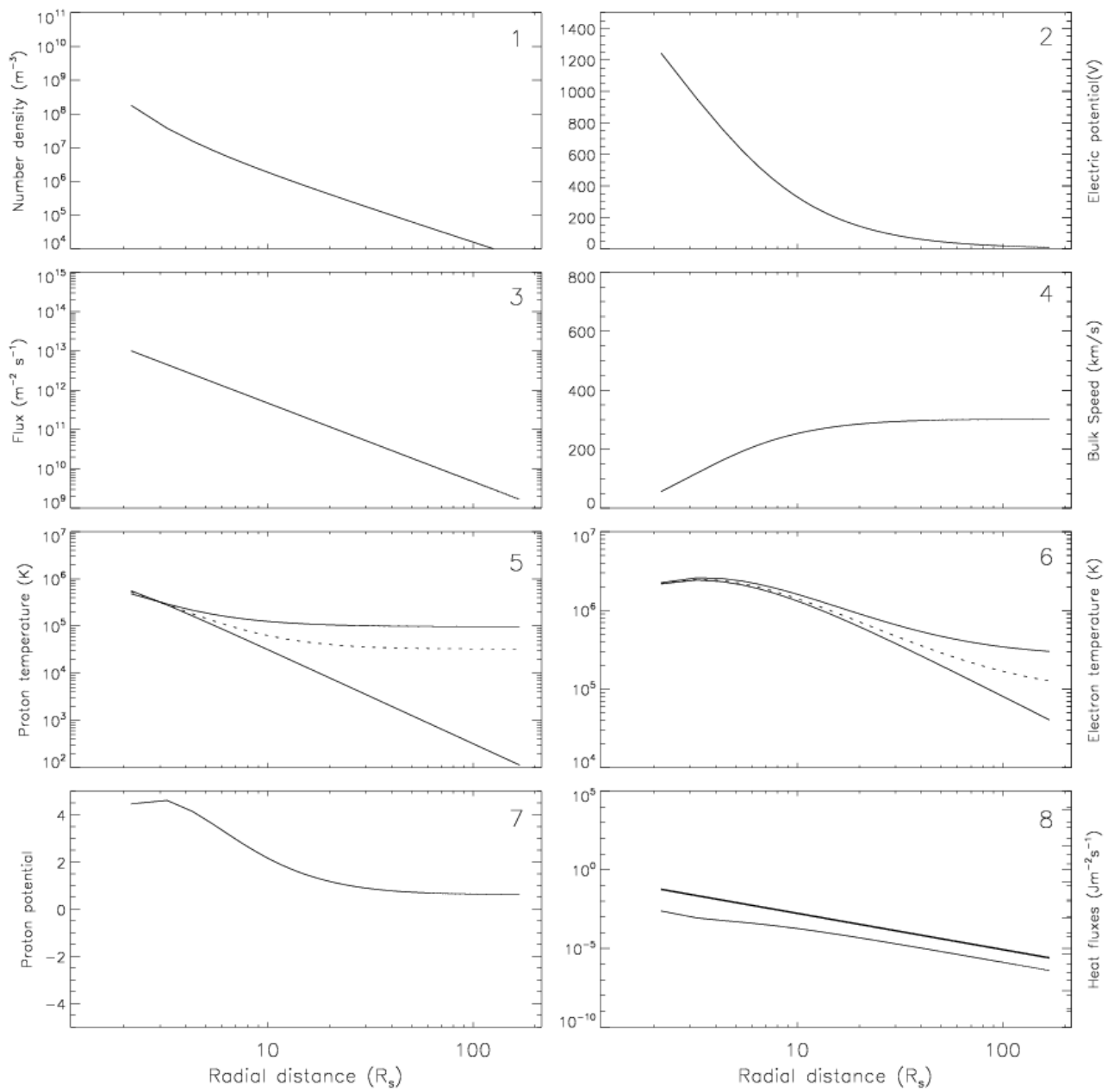

Fig. 3. Profiles of the FDV moments obtained with an exospheric model. Panels 1 to 8 correspond respectively to the density, electric potential, flux, bulk speed, proton temperatures (parallel: upper line, perpendicular: bottom line, average: dotted line), electron temperatures (idem), proton potential and heat flux (electrons: upper line, protons, bottom line).

speed solar wind originating from the coronal holes, are obtained with Kappa between 3 and 4 . The position of the exobase plays also an important role in the solar wind acceleration: the bulk velocity at $1 \mathrm{AU}$ is higher when the exobase is low.

Panels 1 and 3 show the fast decrease of the number density and of the particle flux as a function of the radial distance. Panel 5 and 6 show respectively the proton and electron parallel (upper solid line), perpendicular (bottom solid line) and total (dotted line) temperatures. The electron temperature shows a peak at low radial distance (see panel 6) that is also deduced from coronal observations made during solar eclipses. Simulating a 
coronal hole with a lower value of kappa in the exospheric model, the temperature peak obtained in the solar corona is slightly displaced at higher altitudes, in good agreement again with eclipse observations (Lemaire, 2012).

Note that the profiles of the different moments calculated by the exospheric model are in good agreement with the solar wind observations (Issautier et al., 2001), except for the temperature anisotropy $\mathrm{T}_{/ /} / \mathrm{T}_{\text {perp }}$ which is too high in the model for the electrons as well as for the protons. This is due to the exospheric assumption of the magnetic moment conservation and of the absence of interaction between the particles. The inclusion of a spiral magnetic field reduces the temperature anisotropies at low latitudes (Pierrard et al., 2001a).

The heat flow carried by Kappa distributions in the solar corona is especially high when kappa is small. It does not correspond to the classical Spitzer \& Härm (1953) expression, except when kappa tends to infinity corresponding to the Maxwellian case. Dorelli \& Scudder (1999) demonstrated that a weak power law tail in the electron VDF can allow heat to flow up a radially directed temperature gradient, contrary to the classical heat conduction law. For Kappa distributions, the heat flux is not necessarily proportional to -grad $\mathrm{T}$ (Pierrard, 2011a).

\subsection{The other ions}

Other ions than protons are also present in very low concentration in the solar wind. Protons represent around $90 \%$ of the ions, Helium around $9 \%$, and all the heavier ions less than $1 \%$ all together. They were included in the exospheric models (Pierrard et al., 2004). Their study is very interesting in such models including only the external forces because the ions have different masses and different charges leading to different bulk velocities for each species.

In the exospheric models, the electrostatic potential is determined by the dominant species (electrons and protons). Minor ions have no significant effect on the electrostatic potential, but they are accelerated by this potential at large radial distance since they have a positive charge (like the protons). So they have also a non monotonic potential energy that shows a maximum at low radial distances. An example is illustrated on Fig. 4 illustrating the results of an exospheric model including Helium $\mathrm{He}^{2+}$ and Oxygen ions $\mathrm{O}^{6+}$.

Figure 4 illustrates the profiles of the number density, bulk speed, temperatures (parallel and perpendicular) and the total potential (gravitational and electrostatic) obtained with an exospheric model assuming an exobase at $2 \mathrm{Rs}, \kappa=3$ for the electrons, $\mathrm{T}_{\mathrm{p}}=\mathrm{T}_{\mathrm{e}}=10^{6} \mathrm{~K}, \mathrm{~T}_{\mathrm{He}}=5.10^{7}$ $\mathrm{K}$ and $\mathrm{T}_{\mathrm{O}}=210^{8} \mathrm{~K}$. The $\mathrm{H}^{+}$ions are illustrated by the solid black line, $\mathrm{He}^{2+}$ by the magenta dashed-dotted line and $\mathrm{O}^{6+}$ by the green three dots-dashed line.

The maximum of the total potential is located at slightly larger radial distances for heavier ions since their mass on charge ratio is larger than that of the protons (see panel 4 of Fig. 4). The mass of the particles directly influences the gravitational attraction while the charge determines the electric force. Because the mass and level of ionization is different for each ion species, the bulk velocity is also different for each ion species.

The mass of the minor ions is very high so that they are more difficult to accelerate at high bulk velocities. Nevertheless, these heavy ions can reach high bulk velocities at 1 AU (see panel 2) if their temperature at the exobase is assumed to be sufficiently high (Pierrard et al., 2004). Very high ion temperatures in the solar corona are not unrealistic: their observations 

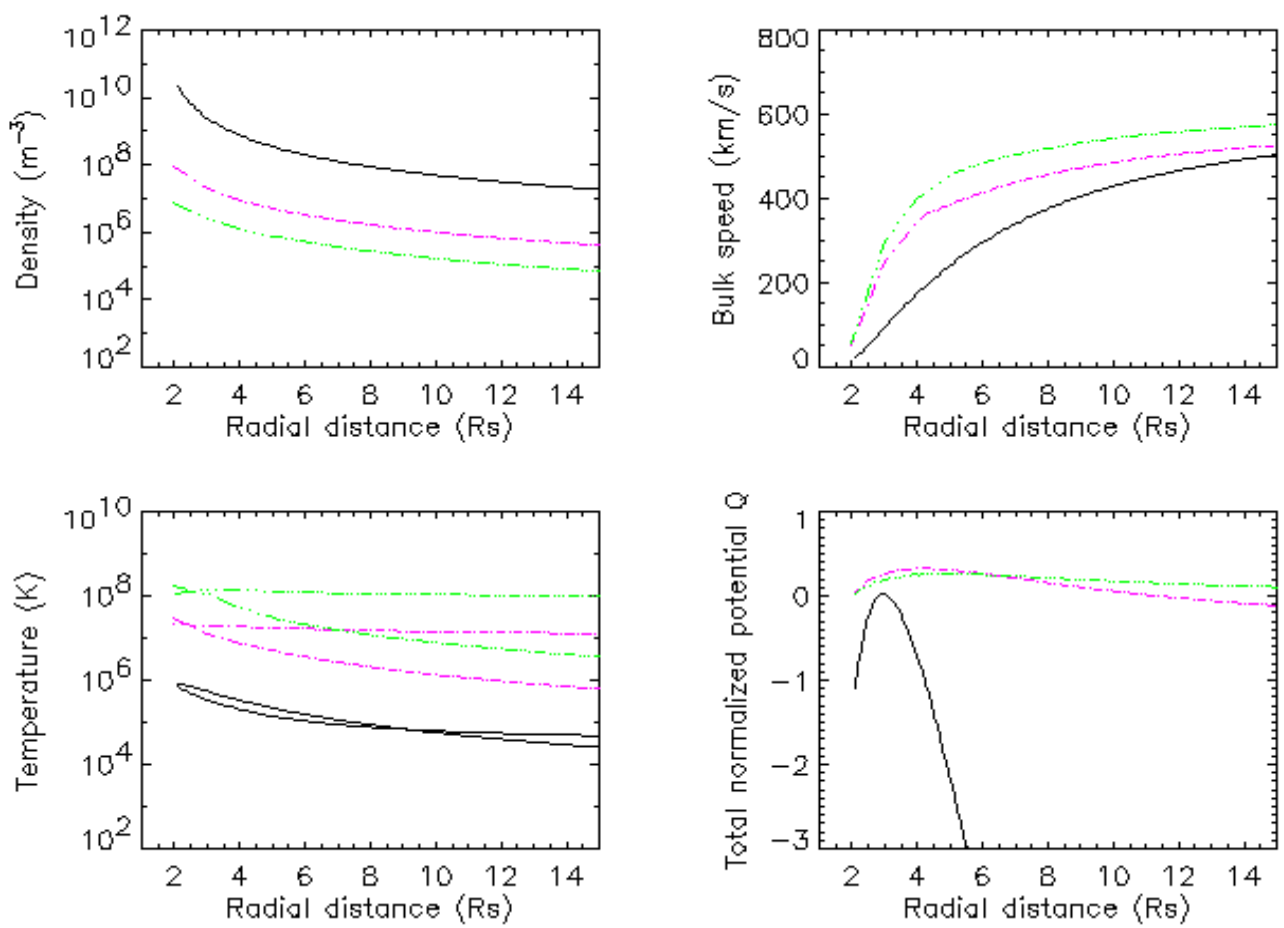

Fig. 4. Profiles of the FDV moments obtained with an exospheric model assuming an exobase at $2 \mathrm{Rs}, \mathrm{\kappa}=3$ for the electrons, $\mathrm{T}_{\mathrm{p}}=\mathrm{T}_{\mathrm{e}}=10^{6} \mathrm{~K}, \mathrm{~T}_{\mathrm{He}}=5.10^{7} \mathrm{~K}$ and $\mathrm{T}_{\mathrm{O}}=210^{8} \mathrm{~K}$. The moments of the $\mathrm{H}^{+}$ions are represented by the solid black line, $\mathrm{He}^{2+}$ by the magenta dasheddotted line and $\mathrm{O}^{6+}$ by the green three dots-dashed line.

deduced from spectroscopy give indeed very high values. Only some ion species have temperatures that can be observed in the solar corona. It is the case of $\mathrm{O}^{5+}$ for which a temperature of the order of $10^{8} \mathrm{~K}$ at $2 \mathrm{Rs}$ is estimated from SUMER on SOHO (Esser \& Edgar, 2000).

The number density decreases in the same proportion for the different particle species independently on their mass or charge, so that the composition of the solar wind remains almost the same in the solar wind as in the corona in the exospheric models (see panel 1).

The perpendicular temperature becomes always lower than the parallel temperature at large radial distances (see panel 3), but this anisotropy can be reduced by assuming large $\mathrm{T}_{\text {perp }} / \mathrm{T} / /$ in the solar corona as it is observed by SUMER at least for oxygen ions and protons (Esser \& Edgar, 2000). The inclusion of the particle interactions such as the Coulomb collisions can also lead to a lower anisotropy. The effects of particle interactions are discussed in the next section.

Different processes have been suggested to explain the very high ion temperatures observed in the solar wind and the corona. Among others, the velocity filtration effect, as suggested 
initially by Scudder (1992), is an interesting possibility: the presence of an enhanced population of suprathermal particles leads to ion temperatures more than proportional to their mass and to an anticorrelation between the temperature and the density of the plasma. Pierrard \& Lamy (2003) showed with a model of the solar corona in hydrostatic equilibrium that VDFs of particles characterized by an enhancement of suprathermal particles lead to a filtration effect that predicts a large increase of the electron and ion coronal temperatures. The temperatures of the ions are more than proportional to their mass, with a small correction depending on their charge state.

Solar wind ion distribution functions have been measured by WIND for $\mathrm{Ne}, \mathrm{O}$ and $\mathrm{He}$ (Collier et al., 1996). Such ion distributions are observed to be characterized by suprathermal tails like in many space plasmas. They have been fitted by Kappa functions with the best kappa parameters as low as $\kappa=2$. Moreover, the temperatures of the ions measured in situ in the high speed solar wind are observed to be more than proportional to their mass, in good agreement with the assumptions made in the model.

\section{The interaction term}

In exospheric models, we considered only the effects of the external forces. We have shown that the averaged values of the moments correspond quite well to what is observed in the solar wind. Nevertheless, the temperature anisotropies are too high and the truncated VDFs are quite different from the solar wind VDF observed in situ. The inclusion of the different interaction processes can help to better model the solar wind and obtain more realistic VDF for the solar particles.

\subsection{The Fokker-Planck Coulomb collision term}

The solar wind is neither a collision-dominated plasma nor a completely collisionless one. This means that neither the hydrodynamic approach, based on the Euler or Navier-Stokes approximations, nor the exospheric or pure collisionless approach are truly appropriate to model the global expansion of the solar wind.

To describe the expansion of the plasma flow out of the hot solar corona, the most appropriate way is to use the kinetic approach since the kinetic processes prevail in the solar corona and solar wind low density plasmas (Marsch, 2006). One has to solve the FokkerPlanck equation which describes the evolution of the VDFs of the particles with the radial distance. The solution of the Fokker-Planck equation is especially significant in the collisional transition region where the Coulomb collisions effects become less and less important with increasing height.

Different models have been developed to study the steady state electron VDF in the corona and at larger radial distances in the solar wind by solving the Fokker-Planck equation (Echim et al., 2011 for a review). Lie-Svendsen et al. (1997) and Lie-Svendsen \& Leer (2000) solved the Fokker-Planck equation using a finite-difference scheme. Landi \& Pantellini (2001, 2003) developed direct particle simulations with binary collisions but the high computational load constrained the mass ratio to be very high $\left(\mathrm{m}_{\mathrm{i}} / \mathrm{m}_{\mathrm{e}}=400\right)$. Recent results of electron VDF simulated using this model are provided in Landi et al. (2010) to study the effects of Coulomb collisions beyond 0.3 AU. 
The most sophisticated collisional model regarding the self-consistent collision term was developed by Pierrard et al. (1999) and Pierrard et al. (2001c). They considered binary Coulomb collisions with electrons and protons using the Fokker-Planck collision operator given by Hinton (1983):

$$
\left(\frac{d f}{d t}\right)_{c}=-\frac{\partial}{\partial \vec{v}} \cdot\left[\vec{A} f-\frac{1}{2} \frac{\partial}{\partial \vec{v}} \cdot(\vec{D} f)\right]
$$

where $\vec{A}$ is the dynamic friction vector and $\vec{D}$ is the velocity diffusion tensor. These terms are given by Rosenbluth et al. (1957) or Delcroix \& Pers (1994) in spherical coordinates.

Pierrard et al. (1999) solved the Fokker-Planck equation using a spectral method where the VDF is expanded in polynomials in velocity, pitch angle and space:

$$
f(x, \mu, z)=\exp \left(-x^{2}\right)\left(\sum_{l=0}^{n-1} \sum_{s=0}^{N-1} \sum_{m=0}^{M-1} a_{l s m} P_{l}(\mu) S_{s}(x) L_{m}(z)\right)
$$

where $x$ is the dimensionless velocity normalized by the thermal velocity, $\mu$ is the cosinus of the pitch angle between the velocity vector and the magnetic field direction, $\mathrm{z}$ is the radial distance, $P_{1}(\mu)$ are Legendre polynomials, $S_{s}(x)$ are speed polynomials and $L_{m}(z)$ are displaced Legendre polynomials.

This spectral method is described in detail in Pierrard (2011b). It was developed and applied previously for the polar wind escaping from the terrestrial atmosphere (Pierrard et al., 1998).

The test electrons are submitted to the influence of the external forces (gravitation, electric and Lorentz forces) and collide with background particles. The VDF of the background electrons was assumed to be the same as the VDF of the test electrons with an iterative process. So, the self collisions are treated consistently by matching the velocity distribution functions of the test and background proton distributions using this iterative numerical method.

The Fokker-Planck model shows the transformation of the electron VDFs in the transition region between the collision-dominated region in the corona and the collisionless region at larger radial distances. While VDF observed by WIND at 1 AU was used as boundary conditions in Pierrard et al. (1999), Pierrard et al. (2001c) have chosen to focus on the region between 2 and 14 solar radii where the Coulomb collisions have the most important effects. The upper limit (14 Rs) corresponds to the region where the mean free path of the particles becomes larger than the density scale height. This range of distance is also interesting because both fluid and exospheric models place the acceleration region of the solar wind at low radial distances from the Sun.

Figure 5 illustrates the electron VDF obtained at 13 Rs with the Fokker-Planck model assuming exospheric conditions at the top of the transition region, i.e., that electrons above a certain escape velocity do not return. Due to this condition, the VDF obtained with the Fokker-Planck model is anisotropic and different from a displaced Maxwellian as used in the Euler (five moments) fluid approximation. The VDF is close to an isotropic distribution at low radial distances and become more and more anisotropic in the transition region. The solar wind model based on the solution of the Fokker-Planck equation produces a core close 
to a Maxwellian due to Coulomb collisions. The halo component due to the enhancement of suprathermal electrons is reproduced only if it is assumed in the boundary conditions. In this case, the halo component increases with the radial distance (Pierrard et al., 1999). The $\mathrm{VDF}$ is also aligned to the magnetic field due to the mirror force, forming the strahl component (Pierrard et al., 2001b).

$$
\text { Velocity distribution funotion }
$$

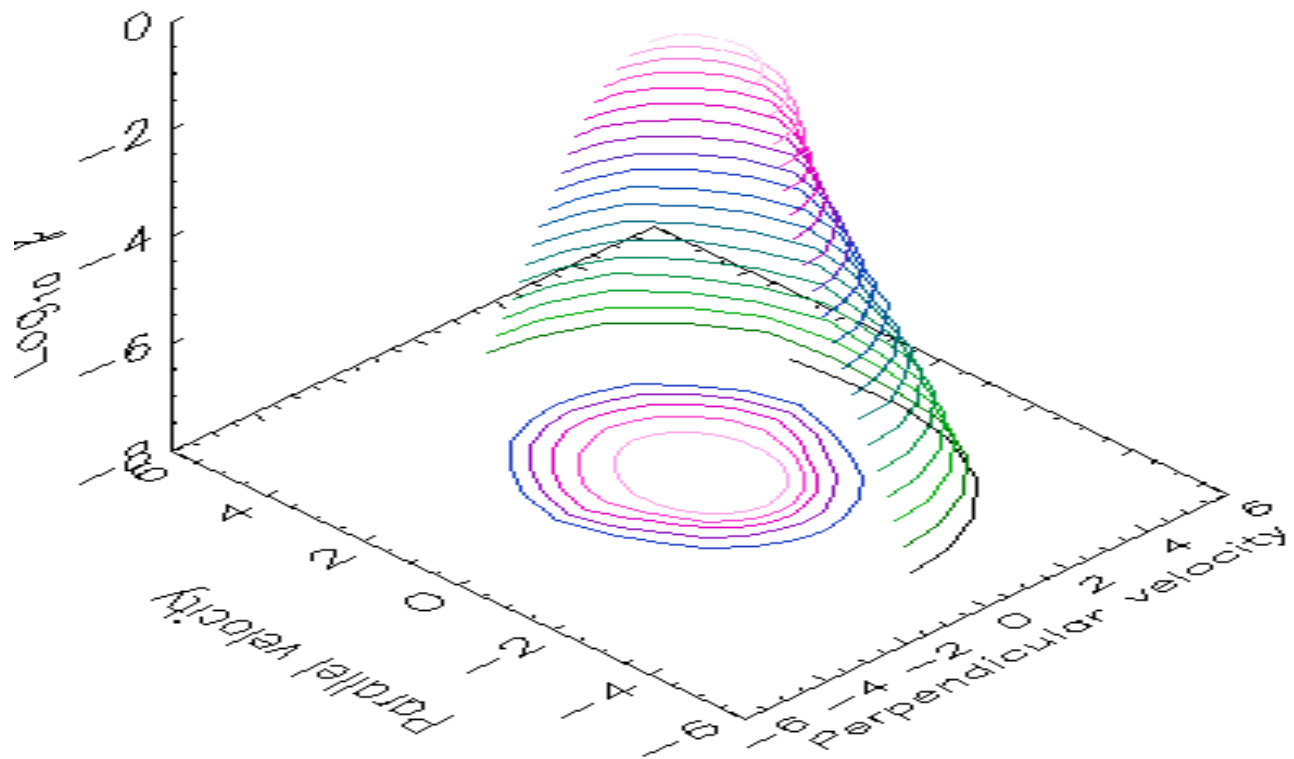

Fig. 5. Electron VDF obtained at 13 Rs with the Fokker-Planck model assuming exospheric conditions at the top of the transition region between collision-dominated and collisionless regions.

It is found that Coulomb collisions have important effects on angular scattering (i.e., on the pitch angle distribution of the electrons) without changing their average density and mean temperatures radial distributions. The boundary conditions have important effects on the solution of the Fokker-Planck equation.

Effects of the Coulomb collisions on the ion and proton VDF were also investigated by Marsch \& Goldstein (1983) and Livi \& Marsch, (1987). These authors showed that in the collisional regions like low-speed wind near the heliospheric current sheet, the Coulomb collisions can maintain an isotropic core, but an escaping tail is obtained in the major part of the high speed solar wind that can be considered as collisionless. It has been proposed that the formation of proton beams in the acceleration region at heliocentric distances 1.1-3 solar radii can be explained by the proton collisional runaway, or by the mirror force acting on the forward part of the proton velocity distribution. 
Even if the inclusion of Coulomb collisions improves the temperature anisotropies and the global shape of the solar particles distributions, significant differences are still obtained between the VDF resulting from the collisional models and the VDF observed in the solar wind. The presence of halo electrons and of proton VDF with an anisotropic core and a beam aligned to the magnetic field remain difficult to explain considering only the external forces and the Coulomb collisions. This shows that other mechanisms exist and have to be taken into account.

\subsection{The whistler turbulence for the electrons}

Resonant interaction with whistler waves in the solar corona and the solar wind was suggested by Vocks \& Mann (2003) and Vocks et al. (2008) to explain the generation of suprathermal electrons. Introducing antisunward-propagating whistler waves into a kinetic model in order to provide diffusion, Vocks et al. (2005) have shown that the whistler waves are capable of influencing the solar wind electron. Vocks (2011) developed a kinetic model for whistler wave scattering of electrons in the solar corona and wind.

Pierrard et al. (2011) have also recently considered the wave-particle resonant interactions in the plasma wave turbulence. These authors showed that the turbulent scattering mean free path is lower than the Coulomb collision mean free path in the solar wind, so that the turbulence mechanism is dominant compared to the Coulomb collisions at sufficiently large radial distances. The quasi-linear wave-particle scattering is again described by the FokkerPlanck equation, using the appropriate diffusion coefficients. They used the Fokker-Planck term determined by Schlickeiser (1989) in the presence of wave turbulence:

$$
\left(\frac{d f}{d t}\right)_{w p}=\frac{\partial}{\partial \mu}\left(D_{\mu \mu} \frac{\partial f}{\partial \mu}+D_{\mu p} \frac{\partial f}{\partial p}\right)+\frac{1}{p^{2}} \frac{\partial}{\partial p} p^{2}\left(D_{p \mu} \frac{\partial f}{\partial \mu}+D_{p p} \frac{\partial f}{\partial p}\right)
$$

where $\mathrm{p}$ is the particle's momentum and the diffusion coefficients are given by Steinacker \& Miller (1992). The electrons are assumed interacting with right-handed polarized waves in the whistler regime. They are considered in uniform fields and a superposed turbulent whistler wave spectrum. Only the slab modes propagating parallel to the interplanetary magnetic field are invoked because the energy exchange with oblique waves is expected to be less significant.

Figure 6 illustrates the electron VDF obtained at 190 Rs with the model including the whistler turbulence and using a typical electron VDF observed at 1 AU by WIND as boundary condition. An increase of the parallel temperature shows that turbulence can reduce the temperature anisotropy found in previous models neglecting this effect. Pierrard et al. (2011) found also that the acceleration of electrons in the solar wind remains mainly due to the electrostatic potential like in exospheric models. Nevertheless, wave turbulence determines the electron pitch-angle diffusion and can be responsible of the formation of the suprathermal tails observed in the solar wind. This is in good agreement with the results of Shizgal (2007) who showed that the VDF tends to Maxwellian only in presence of Coulomb collisions and in the absence of wave-particle interactions. When wave-particle interactions are included, an initial distribution tends to a steady state VDF with nonequilibrium (non-Maxwellian) tails and associated to an increase of the entropy (Leubner, 2004). The role of parallel whistlers can also extend to small altitudes in the acceleration region of the outer corona, where they may explain the energization and the presence of suprathermal electrons. 

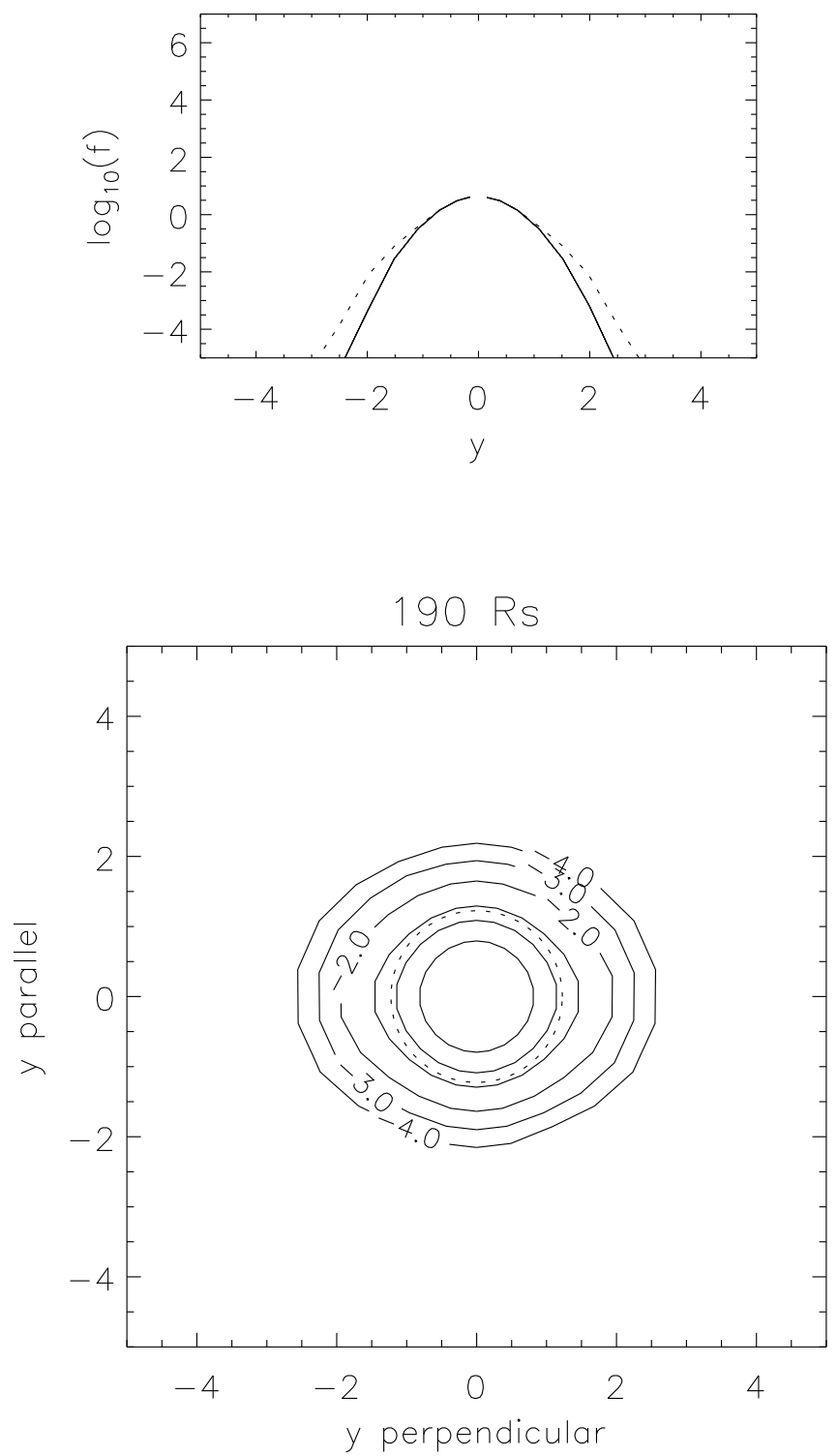

Fig. 6. Electron VDF obtained at 190 Rs with the model including the whistler turbulence (Pierrard et al., 2011).

\subsection{Kinetic Alfven waves for the formation of the proton beam}

Typical proton VDFs observed in the solar wind are characterized by an anisotropic core with $\mathrm{T}_{\text {perp }}>\mathrm{T}_{\backslash \backslash}$ and a proton beam aligned with the magnetic field direction (Marsch et al. 1982). Neither exospheric nor collisional Fokker-Planck models can fully explain these 
characteristics. In view of kinetic Alfven waves (KAW) activity observed in the solar wind, Pierrard \& Voitenko (2010) proposed KAW to play a crucial role for the proton beam formation.

Recent investigations suggest that the wave-particle interactions in the solar wind can be dominated by the waves with short cross-field wavelengths, i.e. in the wavelength range of kinetic Alfven waves (Howes 2008, and references therein).

The generation mechanism for proton beams based on the proton trapping and acceleration by KAWs implies a flux of MHD Alfven waves converting into KAWs linearly and/or nonlinearly. When the proton gyroradius/cross-field wavelengths ratio becomes sufficiently small, the parallel potential well carried by the KAWs traps a fraction of core protons and accelerates them along the background magnetic field. This is illustrated in Fig. 7. The acceleration of trapped protons is caused by the accelerated KAW propagation under the condition that the normalized cross-field wave vector increases. There are several mechanisms in the solar wind, which can increase perpendicular wave number, like phase mixing in shear plasma flows, or in cross-field plasma inhomogeneities or turbulent cascades.

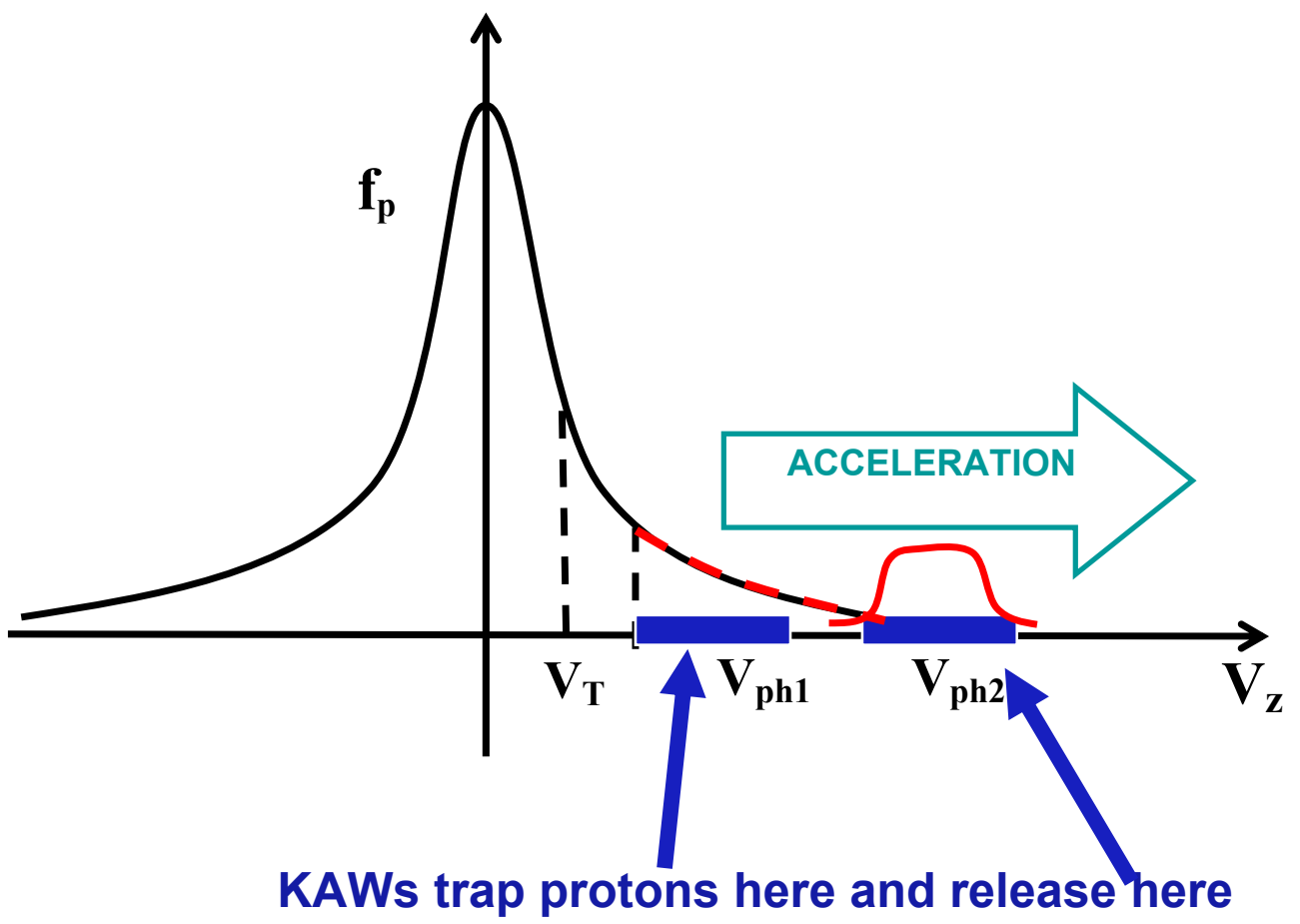

Fig. 7. Illustration of proton VDF obtained with proton trapping and acceleration by KAWs (Pierrard \& Voitenko, 2010). 
The interaction term that is used to simulate the quasi-linear Fokker-Planck diffusion due to kinetic Alfven wave turbulence is given by (Pierrard \& Voitenko 2012):

$$
\left(\frac{d f}{d t}\right)_{Q L}=\frac{\partial}{\partial v}\left(D_{2 D}^{Q L}\right) \frac{\partial f}{\partial v}
$$

where the proton diffusion coefficient is

$$
D_{2 D}^{Q L}=\frac{\pi q^{2}}{2 m^{2}} \sum_{k} \delta\left(\omega_{k}-k_{/ /} v\right) J_{0}\left(k_{\perp} \rho \frac{v_{\perp}}{v_{T}}\right)|E|^{2}
$$

where $\mathrm{q}$ and $\mathrm{m}$ are respectively the charge and the mass of the proton, $\mathrm{k}_{/ /}$and $k_{\perp}$ are the parallel and perpendicular components of the wave vector, $\rho$ is the gyroradius, $v_{T}$ is the thermal velocity, $\delta$ is the Dirac's delta function due to the resonant character of the waveparticle interaction, $\mathrm{J}_{0}$ is the zero-order Bessel function and $\mathrm{E}$ is the parallel component of the electric field.

The model implies a flux of MHD Alfven waves converting into KAWs linearly and/or nonlinearly. When the proton gyroradius/cross-field wavelengths ratio becomes sufficiently small, the parallel potential well carried by the KAWs traps a fraction of core protons and accelerates them along the background magnetic field.

\subsection{Other interactions}

There are also several alternative possibilities to explain the origin of the proton beams (Tu \& Marsch, 2002 and references therein). They can be injected in the solar wind by magnetic reconnection events at the coronal base. The beams can also be produced gradually by the evolution of the proton velocity distributions under the action of wave-particle interactions in the extended region in the solar wind from the acceleration region to $r>0.3$ AU where they are observed. Tu \& Marsch (2002) proposed also a mechanism for the proton beam formation based on the proton-cyclotron resonant interaction with cyclotron modes that can exist in the presence of alpha particles drifting with Alfven velocity.

Araneda et al. (2009) have studied parametric instabilities driven by Alfven-cyclotron waves and their influence on solar wind ions. It was shown that product waves generated by these instabilities can lead to a selective heating and acceleration of different ion species, generating in particular a proton beam with drift speed of about the Alfven speed. However, as there are pros and contras against all mentioned above mechanisms, there is no definite consensus about the physical mechanism producing proton beams.

KAW were also suggested as a possible source for the strong heating of ions across the magnetic field in the solar corona (Voitenko \& Goossens, 2004). Cranmer (2002) has also studied different processes suggested to explain the very high ion temperatures in the corona, and specifically the ion-cyclotron waves with short wavelengths along the background magnetic field. Isenberg et al. (2010b) explained the energization of minor ions in the coronal holes by multiple cyclotron resonances in the presence of dispersive ion cyclotron waves. Solar coronal heating by Alfven wave turbulence was proposed by Bigot et al. (2010). 
Wave-particles interactions are in any case supposed to be important in the evolution of solar wind ion distribution functions (Matteini et al., 2010). The quasilinear effects of resonant wave-particle interaction under condition of imbalanced turbulent heating in the collisionless coronal hole were investigated by Isenberg et al. (2010a). Other instabilities can also be taken into account, like the interplay between Weibel and firehose instabilities in coronal and solar wind outflows (Lazar et al., 2010).

\section{Conclusion}

The acceleration of the solar wind particles can be modeled using the hydrodynamic or the kinetic representations (Parker, 2010). The kinetic approach permits to analyze the effect of each physical process separately. The exospheric models emphasize the effects of the external forces, and especially the effect of the electrostatic potential that accelerates the solar wind particles outwards. Even if such models give already good average values of the solar wind lower order moments, the variety of particle velocity distributions observed in the solar wind cannot be explained by only one mechanism. By including different interaction terms such as Coulomb collisions and wave-particle interactions in the kinetic evolution equation, we can determine their effects on the VDF of the solar wind particles.

Kinetic plasma models including Coulomb collisions with proper boundary conditions are capable in reproducing solar wind speeds, number densities and temperatures compatible with observations. Whistler turbulence can explain the halo suprathermal population of electrons observed in the solar wind VDF. Salient features such as core temperature anisotropies and ion beams propagating with super-Alfven velocities require an additional energy source. An obvious source for that is provided by Alfven waves carrying energy fluxes enough for additional cross-field ion heating and beam production. A new mechanism for the proton beam production via proton trapping and acceleration by KAWs was proposed.

\section{Acknowledgment}

The research leading to these results has received funding from the European Commission's Seventh Framework Program (FP7/2007-2013) inside the grant agreement SWIFF (project $n^{\circ} 2633430$, www.swiff.eu). V. Pierrard thanks the STCE (Solar-Terrestrial Center of Excellence) and BISA for their support.

\section{References}

Araneda, J. A.; Maneva, Y. \& Marsch, E. (2009). Preferential heating and acceleration of alpha particles by Alfven-cyclotron waves. Phys Rev Lett., 102(17):175001, PMID: 19518788

Bigot, B.; Galtier, S. \& Politano, H. (2010). Solar coronal heating via Alvfen wave turbulence. American institute of Physics, AIP Conference Proceedings Solar Wind 12, Volume 1216, pp.48-51 
Collier, M. D.; Hamilton, D. C.; Gloeckler, G.; Boschler, P. \& Sheldon, R. B. (1996). Neon-20, Oxygen-16, and Helium 4 densities, temperatures, and suprathermal tails in the solar wind determined by WIND/MASS. Geophys. Res. Lett., 23, 1191-1194

Cranmer, S. R. (2002). Coronal holes and the high-speed solar wind. Space Sci. Rev., 101, 229294

Delcroix, J.-L. \& Pers, A. (1994). Physique des plasmas 2, Savoirs Actuels, InterEditions/CNRS Editions, Paris, 499p.

Dorelli, J. C. \& Scudder, J. D. (1999). Electron heat flow carried by Kappa distributions in the solar corona. Geophys. Res. Lett., 23, 3537-3540

Echim, M.; Lemaire, J.\& Lie-Svendsen, O. (2011). A Review on Solar Wind Modeling: Kinetic and Fluid Aspects. Surveys in Geophysics, 32 (1), pp. 1-70

Esser, R. \& Edgar, R. J. (2000). Reconciling spectroscopic electron temperature measurements in the solar corona with in-situ charge state observations. Astroph. J. Lett., 532, L71-74

Hinton, F. L. (1983). Collisional transport in plasma, in Basic Plasma Physics I and II, A. A. Galeev and R. N. Sudan (Ed.), North Holland, New York, pp. 148-200

Howes, G. G.; Cowley, S. C.; Dorland, W.; Hammett, G. W; Quataert, E. \& Schekochihin, A. A. (2008). A Model of Turbulence in Magnetized Plasmas: Implications for the Dissipation Range in the Solar Wind. J. Geophys. Res., 113, CiteID A05103

Isenberg, P. A.; Vasquez, B. J.; Chandran B. D. G. \& Pongkitiwanichakul, P. (2010a). Quasilinear wave "reflection" due to proton heating by an imbalanced turbulent cascade. American institute of Physics, AIP Conference Proceedings Solar Wind 12, Volume 1216, pp. 64-67

Isenberg, P. A.; Vasquez, B. J. \& Cranmer, S. R. (2010b). Modeling the preferential acceleration and heating of coronal holes O5+ as measured by UVCS/SOHO, American institute of Physics, AIP Conference Proceedings Solar Wind 12, Volume 1216, pp. 56-59

Issautier, K.; Meyer-Vernet, N.; Pierrard, V. \& Lemaire, J. (2001). Electron temperature in the solar wind from a kinetic collisionless model: application to high-latitude Ulysses observations, Astrophys. Space Sci., 277, 2, 189-193

Lamy, H.; Pierrard, V.; Maksimovic, M. \& Lemaire, J. (2003). A kinetic exospheric model of the solar wind with a non monotonic potential energy for the protons. J. Geophys. Res., 108, 1047-1057

Landi, S., \& Pantellini, F. G. E. (2001). On the temperature profile and heat flux in the solar corona: Kinetic simulations, Astronomy and Astrophys., 372, 686-701

Landi, S., \& Pantellini, F. G. E. (2003) Kinetic simulations of the solar wind from the subsonic to the supersonic regime., Astronomy and Astrophys., 400, 769-778

Landi, S.; Pantellini, F. \& Matteini, L. (2010). Radial evolution of the electron velocity distribution in the heliosphere: role of the collisions. American institute of Physics, AIP Conference Proceedings Solar Wind 12, Volume 1216, pp.218-221

Lazar, M.; Poedts, S. \& Schlickeiser, R. (2010). Nonresonant electromagnetic instabilities in space plasmas: interplay of Weibel and firehose instability. American institute of Physics, AIP Conference Proceedings Solar Wind 12, Volume 1216, pp. 280-283 
Lemaire, J. F. (2012). Determination of coronal temperatures from electron density profiles. Submitted to Solar Physics

Lemaire, J. \& Pierrard, V. (2001). Kinetic models of solar and polar winds. Astrophys. Space Sci., 277, 2, 169-180

Leubner, M. P. (2004). Core-halo distribution functions: a natural equilibrium state in generalized thermostatistics. The Astrophys. J., 604, 469-478

Lie-Svendsen, O.; Hansteen, V. H. \& Leer, E. (1997). Kinetic electrons in high-speed solar wind streams: formation of high-energy tails. J. Geophys. Res., 102, 4701

Lie-Svendsen, O. \& Leer, E. (2000). The electron velocity distribution in the high-speed solar wind: Modeling the effects of protons. J. Geophys. Res., 105, 35-46

Livi, S. \& Marsch, E. (1987). Generation of solar wind proton tails and double beams by Coulomb collisions. J. Geophys. Res, 92, 7255

Maksimovic, M.; Pierrard, V. \& Lemaire, J. (1997a). A kinetic model of the solar wind with Kappa distributions in the corona. Astron. Astrophys., 324, 725-734

Maksimovic, M.; Pierrard, V. \& Lemaire, J. (2001). On the exospheric approach for the solar wind acceleration. Astrophys. Space Sci., 277, 2, 181-187

Maksimovic, M., Pierrard, V. \& Riley, P. (1997b) Ulysses electron distributions fitted with Kappa functions. Geophys. Res. Let., 24, 9, 1151-1154

Marsch, E. (2006). Kinetic physics of the solar corona and the solar wind, Living Rev. Solar Phys., 3, 1 (www.livingreviews.org/lrsp-2006-1)

Marsch, E. \& Goldstein, H. (1983). The effects of Coulomb collisions on solar wind ion velocity distributions. J. Geophys. Res., 88, A12, 9933-9940

Marsch, E.; Muehlhauser, K.-H.; Schwenn, R.; Rosenbauer, H.; Pilipp, W. \& Neubauer, F. (1982). Solar wind protons: Three-dimensional velocity distributions and derived plasma parameters. J. Geophys. Res., 87, 52-72.

Matteini, L.; Lansi, S.; Velli, M. \& Hellinger, P. (2010). On the role of wave-particle interactions in the evolution of solar wind ion distribution functions. American institute of Physics, AIP Conference Proceedings Solar Wind 12, Volume 1216, pp.223226

Parker, E. N. (2010). Kinetic and hydrodynamic representations of coronal expansion and the solar wind. American institute of Physics, AIP Conference Proceedings Solar Wind 12, Volume 1216, pp.3-7

Pierrard, V. (2011a). Solar wind electron transport: interplanetary electric field and heat conduction. Space Science Review (solar wind), doi: 10.1007/s11214-011-9743-6

Pierrard, V. (2011b). A numerical method to determine the particle velocity distribution functions in space. in Astronum2010 Proceedings, Numerical Modeling of Space Plasma Flows, Astronomical Society of the Pacific Conference series, Edited by N. V. Pogorelov, E. Audit and G. P. Zank, vol. 444, 166-176

Pierrard, V.; Issautier, K.; Meyer-Vernet, N. \& Lemaire, J. (2001a). Collisionless solar wind in a spiral magnetic field. Geophys. Res. Lett., 28, 2, 223-226

Pierrard, V. \& Lamy, H. (2003). The effects of the velocity filtration mechanism on the minor ions of the corona. Solar Physics, 216, 47-58 
Pierrard, V.; Lamy, H. \& Lemaire, J. (2004). Exospheric distributions of minor ions in the solar wind. J. Geophys. Res., vol. 109, A2, A02118, p.1-13, doi: 10.1029/2003JA010069

Pierrard, V. \& Lazar, M. (2010). Kappa distributions: theory and applications in space plasmas. Solar Physics, vol. 287, N 1, 153-174, doi: 10.1007/s11207-010-9640-2

Pierrard, V.; Lazar, M. \& Schlickeiser, R. (2011). Evolution of the electron distribution function in the wave turbulence of the solar wind. Solar Phys. 269, 2, 421-438, DOI $10.1007 /$ s11207-010-9700-7

Pierrard, V. \& Lemaire, J. (1996). Lorentzian ion exosphere model. J. Geophys. Res., 101, 79237934

Pierrard, V. \& Lemaire, J. (1998) A collisional kinetic model of the polar wind. J. Geophys. Res., 103, A6, 11701-11709

Pierrard, V.; Maksimovic, M. \& Lemaire, J. (1999). Electron velocity distribution function from the solar wind to the corona. J. Geophys. Res., 104, 17021-17032

Pierrard, V.; Maksimovic, M. \& Lemaire, J. (2001b). Core, halo and strahl electrons in the solar wind. Astrophys. Space Sci., 277, 2, 195-200

Pierrard,V.; Maksimovic, M. \& Lemaire, J. (2001c). Self-consistent kinetic model of solar wind electrons, J. Geophys. Res., 107, A12, 29.305-29.312

Pierrard, V. \& Voitenko, Y. (2010). Velocity distributions and proton beam production in the solar wind. American institute of Physics, AIP Conference Proceedings Solar Wind 12, Volume 1216, pp. 102-105

Pierrard, V. \& Voitenko, Y. (2012). Formation of proton beams due to kinetic Alfven waves in the solar wind. Submitted to Solar Phys.

Rosenbluth, M. N.; McDonald, W. \& D. L. Judd, D. L. (1957). Fokker-Planck equation for an inverse-square force. Phys. Rev., 107, 1

Schlickeiser, R. (1989). Cosmic-ray transport and acceleration. I - Derivation of the kinetic equation and application to cosmic rays in static cold media. II - Cosmic rays in moving cold media with application to diffusive shock wave acceleration. The Astrophysical Journal, Part 1 vol. 336, 243-293, ISSN 0004-637X

Scudder, J. D. (1992). Why all stars possess circumstellar temperature inversions. The Astrophys. J., 398, 319-349

Shizgal, B. D. (2007). Suprathermal particle distributions in space physics: kappa distributions and entropy. Astrophys. Space Sci., 312, 227-237

Spitzer, L. Jr. \& Härm, R. (1953). Transport phenomena in a completely ionized gas. Phys. Rev., vol. 89, 5, 977-981

Steinacker, J. \& Miller, J. A. (1992). Stochastic gyroresonant electron acceleration in a lowbeta plasma. 1. Interaction with parallel transverse cold plasma waves. Astrophys. J., 393, 764-781

Tu, C. Y. \& Marsch, E. (2002). Anisotropy regulation and plateau formation through pitch angle diffusion of solar wind protons in resonance with cyclotron waves. Journal of Geophysical Research-Space Physics, 107, (A9) pp. SSH 8-1, CiteID 1291, DOI 10.1029/2002JA009264

Vocks, C. (2011). Kinetic models for whistler wave scattering of electrons in the solar corona and wind. Space Sci. Rev., doi 10.1007/s11214-011-9749-0 
Vocks, C. \& Mann, G. (2003). Generation of suprathermal electrons by resonant waveparticle interaction in the solar corona and wind. The Astroph. J., 593, 1134-145

Vocks, C.; Salem, C.; Lin, R. P. \& Mann, G. (2005). Electron halo and strahl formation in the solar wind by resonant interaction with whistler waves. The Astrophys. Journal, 627

Vocks, C. \& Mann, G. (2008). Formation of suprathermal electron distributions in the quiet solar corona. Astron. Astroph., 480, 527-536

Voitenko, Y. \& Goossens, M. (2004). Cross-field heating of coronal ions by low-frequency kinetic Alfven waves. The Astrophys. J., 605: L149-L152 


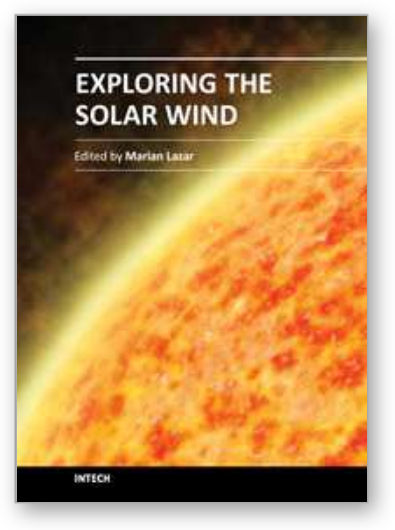

\author{
Exploring the Solar Wind \\ Edited by Dr. Marian Lazar
}

ISBN 978-953-51-0339-4

Hard cover, 462 pages

Publisher InTech

Published online 21, March, 2012

Published in print edition March, 2012

This book consists of a selection of original papers of the leading scientists in the fields of Space and Planetary Physics, Solar and Space Plasma Physics with important contributions to the theory, modeling and experimental techniques of the solar wind exploration. Its purpose is to provide the means for interested readers to become familiar with the current knowledge of the solar wind formation and elemental composition, the interplanetary dynamical evolution and acceleration of the charged plasma particles, and the guiding magnetic field that connects to the magnetospheric field lines and adjusts the effects of the solar wind on Earth. I am convinced that most of the research scientists actively working in these fields will find in this book many new and interesting ideas.

\title{
How to reference
}

In order to correctly reference this scholarly work, feel free to copy and paste the following:

Viviane Pierrard (2012). Kinetic Models of Solar Wind Electrons, Protons and Heavy lons, Exploring the Solar Wind, Dr. Marian Lazar (Ed.), ISBN: 978-953-51-0339-4, InTech, Available from:

http://www.intechopen.com/books/exploring-the-solar-wind/kinetic-models-of-solar-wind-electrons-protonsand-heavy-ions

\section{INTECH}

open science | open minds

\section{InTech Europe}

University Campus STeP Ri

Slavka Krautzeka 83/A

51000 Rijeka, Croatia

Phone: +385 (51) 770447

Fax: +385 (51) 686166

www.intechopen.com

\section{InTech China}

Unit 405, Office Block, Hotel Equatorial Shanghai

No.65, Yan An Road (West), Shanghai, 200040, China

中国上海市延安西路65号上海国际贵都大饭店办公楼405单元

Phone: +86-21-62489820

Fax: +86-21-62489821 
(C) 2012 The Author(s). Licensee IntechOpen. This is an open access article distributed under the terms of the Creative Commons Attribution 3.0 License, which permits unrestricted use, distribution, and reproduction in any medium, provided the original work is properly cited. 